\title{
Increased prevalence of symptomatic macrovascular disease in systemic sclerosis
}

University Department of Medicine,

Ninewells Hospital, Dundee DD1 9SY, United Kingdom

D J Veale

T A Collidge

J J F Belch

Correspondence to: Dr D J Veale,

University Department

of Medicine

Ninewells Hospital,

Ninewells Hospital,

United Kingdom.

Accepted for publication

8 June 1995

D J Veale, T A Collidge, J J F Belch

\begin{abstract}
Objectives-To determine the prevalence of symptomatic macrovascular disease, as defined by the World Health Organisation questionnaire for intermittent claudication, in patients with systemic sclerosis (SSc), and to compare the prevalence in this population with a cross sectional population study group reported in the Edinburgh Artery Study (EAS).

Methods-A group of 53 patients with a diagnosis of limited or diffuse systemic sclerosis were identified from our register. They were asked to complete the WHO questionnaire to establish the presence or absence of intermittent claudication. In addition, each patient's case notes were reviewed to establish the existence of definite peripheral vascular disease and to determine their risk factor profile.

Results-Forty six patients responded to the questionnaire, giving a response rate of $87 \%$. Intermittent claudication was found in $10 \mathrm{SSc}$ patients $(21 \cdot 7 \%)$ according to the questionnaire (two with diffuse and eight with limited SSc), compared with a prevalence rate of $4.6 \%$ for claudication in the EAS. Three SSc patients experienced clinical events attributable to occlusion of a major artery proven on angiography. Four patients had hypertension, there were three current and four ex-smokers, and two had increased total cholesterol. None of these patients had diabetes.

Conclusions-This study demonstrated a greater prevalence of macrovascular disease in patients with SSc than had been found in a neighbouring population.
\end{abstract}

(Ann Rheum Dis 1995; 54: 853-855)

Systemic sclerosis (SSc) is characterised by a complex fibrotic lesion of connective tissues, and changes in the vascular bed including intimal proliferation and fibrous thickening of the media ${ }^{1}$ are described. Raynaud's phenomenon is also a common problem in SSc. ${ }^{2}$ Several possible mechanisms may be responsible for ischaemia in SSc, including vasospasm, ${ }^{3}$ endothelial damage,${ }^{4}$ or abnormalities of haemostasis. ${ }^{5}$ The extent of vascular involvement has become more thoroughly defined, and we now know it to include a wide variety of internal organs such as the oesophagus, ${ }^{6}$ the heart, ${ }^{7}$ and the cerebral circulation, ${ }^{8}$ in addition to the peripheries. An association between anticentromere antibody and increased risk of ischaemic digit loss has been reported, ${ }^{9}$ and there have been case reports linking anticentromere and antiphospholipid antibodies to large and medium vessel thrombosis in calcinosis, Raynaud's phenomenon, oesophageal dysmotility, sclerodactyly, telangiectasia (CREST). ${ }^{10-12}$ In addition, there have been only a small number of case reports ${ }^{1314}$ of macrovascular disease in SSc patients, and retrospective postmortem studies $^{1516}$ suggest that macrovascular disease is not a recognised feature of SSc. The prevalence of macrovascular disease in SSc is therefore not known accurately. We reported previously that a similar prevalence of large vessel occlusive events occurred in SSc patients as in the general population. ${ }^{17}$ The aim of this study was to determine in a cross sectional study the prevalence of intermittent claudication in our SSc patients and the risk factor profile of those patients. In addition, the prevalence of coexistent symptomatic disease involving the coronary and cerebral systems was examined.

\section{Patients and methods}

Patients with a diagnosis of SSc currently attending the unit were identified from our register of SSc patients. The diagnosis of SSc was based on the American Rheumatism Association (ARA) criteria. $^{18}$ The patients were classified as having limited or diffuse disease according to clinical features-limited scleroderma of the distal limbs including features of CREST, diffuse SSc defined as proximal scleroderma, or major internal organ involvement. All patients currently attending the clinic were approached and studied in a prospective manner. The World Health Organisation questionnaire ${ }^{19}$ specifically designed to identify subjects with intermittent claudication and validated in a large epidemiological study of a Scottish population (the Edinburgh Artery Study (EAS) ${ }^{20}$ ) was used. The questionnaire was sent to the patients, and if no response was received a further questionnaire was sent after four weeks. Only calf pain specifically caused by exercise and relieved by rest was considered to be intermittent claudication; other types of pain, including musculoskeletal pain, generalised pedal pain, and other causes of pain on walking, were not regarded as positive. Following this, the case notes of all patients were reviewed to establish the presence or absence of coexistent coronary and cerebrovascular disease and to identify risk factors. 


\section{Results}

Fifty three SSc patients were identified and invited to participate. Ten had diffuse SSc and 43 had limited disease. A total of 46 patients (42 women) replied after two questionnaires were sent-a response rate of $87 \%$. The median age was 57 years (range 18-78). The median duration of symptoms was 14 years (range six months to 50 years). Eight of the 46 responders had diffuse SSc and 38 had limited SSc or CREST variant. A positive questionnaire response for intermittent claudication was found in 10 patients $(21 \cdot 7 \%)$. Four claudicants were in the same age group as the EAS patients (55-74), but five were younger than this age range, indicating that the finding of claudication was even more significant. Two of the 10 patients had diffuse SSc and eight had the limited variant. Hypertension was a risk factor in four subjects, smoking in seven (four were ex-smokers), hypercholesterolaemia was present in two individuals, and none of these patients was diabetic.

Seven $(70 \%)$ claudicants had symptomatic ischaemic heart disease (IHD)-current or previous angina or myocardial infarction, compared with seven of $36(19 \cdot 4 \%)$ nonclaudicants. Hypertension was present in four $(40 \%)$ and hypercholesterolaemia in two $(20 \%)$ claudicants, compared with $25 \%$ and $16.6 \%$ of non-claudicants, respectively. Three claudicants $(30 \%)$ had suffered from a previous stroke, compared with $5.5 \%$ of nonclaudicants. The prevalence of smoking was $70 \%$ (including four ex-smokers) in claudicants, almost double that in non-claudicants (38.8\%, including six ex-smokers).

Three patients, including two claudicants, had previously experienced a significant large vessel occlusion requiring angiography and intervention, thus confirming the presence of macrovascular disease. All three patients were smokers; one had high serum cholesterol and symptomatic IHD, while the other two had suffered from transient ischaemic attacks indicating disease of the cerebrovascular system.

\section{Discussion}

This study has found the prevalence of intermittent claudication in SSc to be $21.7 \%$. There was a coexistent prevalence of IHD of $15 \cdot 2 \%$ and of cerebrovascular disease of $6.5 \%$. In nine patients with claudication, at least one associated risk factor for vascular disease such as hypertension, history of smoking, and hypercholesterolaemia was present, although none of these patients was diabetic.

Microvascular changes are well described in $\mathrm{SSc}^{21}$ and associated with symptoms such as Raynaud's phenomenon, digital infarcts, ulceration, and gangrene. The role of antiphospholipid antibodies in large and medium vessel thrombosis in SSc has been noted, ${ }^{10} 11$ and Barile et al reported similar findings without antiphospholipid antibodies. ${ }^{12}$ An association with macrovascular disease has been suggested by a small number of case reports; ${ }^{12-14}$ however, to date there are no prospective studies examining this issue. In pathological studies ${ }^{15} 16$ there has been little convincing evidence of large vessel atherosclerosis; however, one of these papers was a review and the other was a report based on a retrospective postmortem survey from the case notes.

The prevalence of intermittent claudication in a Health Region neighbouring DundeeLothian-estimated the prevalence of intermittent claudication using the WHO questionnaire to be $4.5 \%$, and major asymptomatic disease with significant impairment of blood flow as $8.0 \% .{ }^{20}$ In comparison with these rates, the present study suggests that the prevalence of symptomatic macrovascular disease as defined by intermittent claudication $(21 \cdot 7 \%)$ is much greater in SSc than in the EAS study population. Although postal questionnaires may have limitations, not least that symptoms may be open to interpretation, both studies did involve the same questionnaire. The populations are not directly comparable, as the EAS was a cross sectional age stratified random sample from 10 general practices, and related to a predominantly male population, a decade older than our cohort. Our finding of a greater prevalence of claudication in a population of females younger than the EAS population adds emphasis to the significance of the observation. Direct comparison with a younger, predominantly female group would be more appropriate, though this is likely to increase further the differences between findings of our study and the EAS. To achieve statistical analysis for this comparison it would be necessary to examine more than 200 patients with SSc. A more rational approach would be a case-control study of intermittent claudication in SSc, and such a study has been planned. It is also reasonable to suspect that, as in other studies, the rate of asymptomatic disease will be even greater, and we propose to assess this also.

Four of the claudicants (40\%) were hypertensive, compared with nine $(25 \%)$ of the 36 non-claudicants; ischaemic heart and cerebrovascular disease was more prevalent in the claudicants also. Seven claudicants $(70 \%)$ had a significant smoking history and three patients continued to smoke, whereas $14(38 \cdot 8 \%)$ of the non-claudicants were either smokers or ex-smokers. Two claudicants $(20 \%)$ had increased serum cholesterol levels compared with six $(16 \cdot 6 \%)$ of the non-claudicants. The finding of positive risk factors in greater proportions of the claudicants suggests that it will be important to assess these patients for risk factors with a view to manipulating their risk profile. The presence of anticardiolipin antibodies was not tested in these patients; although uncommon, it may be relevant in some patients with SSc. The patients in our study were not taking corticosteroids, which have been tenuously linked with increased risk for atherosclerosis. It is now clear that, with modern antihypertensive treatments, renal crisis is no longer the major threat to this group of patients and as their survival may be prolonged, greater attention needs to be given to 
problems such as macrovascular disease and its attendant risk factors. The question arises whether we should be screening SSc patients for risk factors and existent macrovascular disease with a view to prophylactic treatment? Should patients with SSc be given aspirin as prophylaxis? Aspirin, in the US Physician's Health Study, has recently been shown to reduce the need for peripheral vascular surgery in apparently healthy men. ${ }^{22}$ However, there is little evidence to suggest that aspirin is beneficial in the treatment of Raynaud's phenomenon associated with SSc, therefore it is not clear if aspirin should be given as a prophylactic agent for macrovascular disease. Our findings from this small study have prompted us to initiate further studies of the prevalence and incidence of macrovascular disease in an SSc population compared with that in an age, gender, and geographically matched general population, and of all risk factors which might assist in the identification of those patients with symptomatic and asymptomatic macrovascular disease.

In conclusion, the results of this preliminary study suggest that patients with SSc have a greater prevalence of intermittent claudication compared with a geographically similar population. This suggests that there is an association between SSc and macrovascular disease. Furthermore, it may be important to screen for other risk factors which may be favourably altered.

1 Medsger T A. In: McCarty D J, ed. Arthritis and allied conditions. Philadelphia: Lea \& Febiger, 1989; 1118-65.

2 Belch J J F. Raynaud's phenomenon: its relevance to scleroderma. Ann Rheum Dis 1991; 50: 839-45.

3 Walmsley D, Goodfield M J D. Evidence for an abnormal peripherally mediated vascular response to temperature
in Raynaud's phenomenon. $B r \mathcal{F}$ Rheumatol 1990; 29: $181-4$.

4 Belch J J F, Zoma A A, Richards I M, McLaughlin K, Forbes C D, Sturrock R D. Vascular damage and Factor VIII related antigen in the rheumatic diseases. Rheumatol Int 1987; 7: 107-11.
5 Lau C S, Veale D, MacLaren $M$, Bancroft A J, Belch J J F. Increased whole blood platelet aggregation in patients with Raynaud's phenomenon with and without patients with Raynaud's phenomenon with and witho
systemic sclerosis. Arthritis Rheum 1992; S35: B211.

6 Belch J J F, Land D, Park R H R, McKillop J. Decreased oesophageal blood flow in patients with Raynaud's phenomenon. BrF Rheumatol 1988; 27: 426-30

7 Kahan A, Devaux J Y, Amor B, et al. Nifedipine and thallium-201 myocardial perfusion in progressive systemic sclerosis. N Engl F Med 1986; 314: 1397-402.

8 Kallenberg C G M, Wouda A A, The T H. Systemic involvement and immunological findings in patients presenting with Raynaud's phenomenon. Am $\mathcal{F}$ Med 1980; 69: 675-80.

9 Wigley F M, Wise R A, Miller R, Needleman B W, Spence $\mathbf{R}$ J. Anticentromere antibody as a predictor of digital loss in patients with systemic sclerosis. Arthritis Rheum 1992; 36: 688-93.

10 Shapiro L S. Large vessel arterial thrombosis in systemic sclerosis associated with antiphospholipid antibodies. f Rheumatol 1990; 17: 685-8.

11 Merino J, Casanueva B, Piney E, Val Bernal F, Rodriguez-Valverde V. Hemiplegia and peripheral gangrene secondary to large and medium size vessels involvement in CREST syndrome. Clin Rheumatol 1982; 1: 295-9.

12 Barile L A, Bravo G, Salvador P. Large vessel arterial thrombosis in systemic sclerosis with antiphospholipid antibodies. $\mathcal{F}$ Rheumatol 1990; 17: 1721.

13 Youssef P, Englebert H, Bertouch J. Large vessel occlussive disease associated with CREST syndrome and scleroderma. Ann Rheum Dis 1993; 52: 464-6.

14 Furey N L, Schmid F R, Kwaan H C, Friederici H H. Arterial thrombosis in scleroderma. Br $\mathcal{F}$ Dermatol 1966; 78: 24-8.

15 Norton W L, Nardo J M. Vascular disease in progressive systemic sclerosis (scleroderma). Ann Int Med 1970; 73: 317-24

16 D'Angelo W A, Fries J F, Masi A T, Shulman L E. Pathologic observations in systemic sclerosis (scleroderma). Am $\mathcal{F}$ Med 1969; 46: 428-39.

17 Veale D, Belch J J F. Large vessel occlusive disease in systemic sclerosis. Ann Rheum Dis 1993; 52: 837-8.

18 Subcommittee for Scleroderma Criteria of the American Rheumatism Association Diagnostic and Therapeutic Criteria Committee (1980). Preliminary criteria for the classification of systemic sclerosis (scleroderma). Arthritis Rheum 1980; 23: 581-90.

19 Rose G A. The diagnosis of ischaemic heart pain and intermitten claudication in field surveys. Bull WHO 1962; 27: 645-58.

20 Fowkes F G, Housley E, Cawood E H, MacIntyre C C, Ruckley C V, Prescott R J. Edinburgh artery study: prevalence of asymptomatic and symptomatic peripheral arterial disease in the general population. Int $\mathcal{f}$ Epidemiol 1991; 20: 384-92.

21 Maricq H R. Raynaud's phenomenon and microvascular abnormalities in scleroderma. In: Jayson M I V, Black C M, eds. Systemic sclerosis: scleroderma. London: J Wiley \& Sons Ltd, 1988; 151-66.

22 Goldhaber S Z, Manson J E, Stampfer M J, et al. Low-dose aspirin and subsequent peripheral arterial surgery in the Physician's Health Study. Lancet 1992; 340: 143-5. 